\title{
Debate sobre los procesos curriculares de las escuelas ubicadas en zonas afectadas por el conflicto armado en Montelíbano (Colombia)
}

\author{
Manco Atencio, Jaime \\ jaimemanco@hotmail.com \\ Facultad de Humanidades y Ciencias de la Educación \\ Universidad Metropolitana de Educación, Ciencia y Tecnología de Panamá
}

\section{RESUMEN}

La configuración e implementación de Modelos Pedagógicos que respondan a las necesidades e intereses de la comunidad en la cual está inmersa es el deber ser de la escuela. Sin embargo, la adaptación de modelos foráneos, ha impedido una adecuada lectura del contexto para la construcción de un Modelo Pedagógico pertinente. El presente artículo pretende analizar los diseños curriculares implementados en las instituciones educativas ubicados en zonas afectas por el conflicto armado en el municipio de Montelíbano Córdoba para describir su incidencia en el desarrollo de la educación rural de la región en el marco del acuerdo final de paz entre el gobierno de Colombia y la FARC-EP. Mediante la revisión documental de los proyectos educativos institucionales y los informes técnicos de visitas para la implementación de la jornada única de las siete instituciones rurales focalizadas por la entidad territorial certificada Córdoba reabriendo en debate de la brecha social entre lo urbano y lo rural, resultando paradójico que las escuelas con mayor afectación por el conflicto armado no cuente con personal cualificado como psicorientadores, etnoeducadores y solamente dos de ellas cuente con convenios para la formación de sus estudiantes en producción agropecuaria y piscicultura.

Palabras clave: Procesos curriculares; modelos pedagógicos; conflicto armado; proceso de paz; Postconflicto. 


\title{
Debate on the curricular processes of schools located in areas affected by the armed conflict in Montelíbano (Colombia)
}

\begin{abstract}
It is the duty of a school to design and implement Pedagogical Models which respond to the needs and interests of the community in which it is immersed. However, the adaptation of foreign models has prevented an adequate understanding of the context which impedes the implementation of a relevant Pedagogical Model. This article aims to analyze curriculum designs implemented in educational institutions in areas affected by the armed conflict in the municipality of Montelíbano, Córdoba with a view to describing their impact on the development of rural education in the region within the framework of the final peace agreement between the government of Colombia and the FARC-EP. Through a documental review of institutional educational projects and technical reports of visits with the seven rural, single-shift institutions in the department of Córdoba, findings include: a need to return to the debate around the urban, rural divide along social lines, a paradoxical finding that the schools most affected by the armed conflict do not have qualified personnel such as psychotherapists and ethno-educators and only two of them have agreements for the training of their students in agricultural production and fish farming.
\end{abstract}

Keywords: Curricular processes; pedagogical models; armed conflict; peace process; Post-conflict.

Artículo recibido: 19 enero 2020

Aceptado para publicación: 22 febrero Correspondencia: jaimemanco@hotmail.com Conflictos de Interés: Ninguna que declarar 


\section{INTRODUCCIÓN}

Los sistemas educativos surgidos a través de la historia han abordado las diferentes concepciones del conocimiento, los métodos, la didáctica, los contenidos, las formas de evaluación, así como la relación entre docentes y estudiante; todos ellos convergen en punto donde la complejidad de la enseñanza y el aprendizaje requiere un diseño curricular igualmente complejo que atienda a las necesidades e intereses de los actores del proceso educativo desde las realidades sociales de la época.

De acuerdo con lo anterior, surge el siguiente interrogante ¿las escuelas se actualmente encuentran preparadas para asumir el reto de responder a las exigencias de la sociedad donde se encuentra inmersa?, hecho que obliga a la reflexión y posterior rediseño (si lo amerita) de sus procesos curriculares, lo que implica transformar las prácticas educativas tanto de los docentes como de los educandos buscando con ello estar a la par con los fenómenos sociales, culturales y políticos de la época que le permita cumplir con su encargo de formar individuos útiles a la sociedad, al mismo tiempo que respete la individualidades, estilos y ritmos de aprendizajes pero sobre todo las necesidades e intereses de los educandos Teniendo en cuenta que desde las épocas más remotas la raza humana se ha visto envuelta en episodios de barbarie y violencia presentes tanto en las antiguas civilizaciones hasta nuestros días, América Latina no ha sido la excepción. Así se puede constatar al remontarse al menos a la historia más reciente desde los tiempos prehispánicos se encuentran las batallas de los Caribes y Taínos en las Antillas menores, la conquista y reconquista española, violencia política, control militar-político-económico de grupos armados al margen de la ley, narcotráfico, desmovilización, en fin un doloroso tránsito por diferentes conflictos y guerras con los que ha tenido que convivir de manera paralela la escuela; que lamentablemente en palabras de Latorre Restrepo, S. (2018), "hoy es imposible pensar en un escenario de posconflicto cuando todavía las estructuras paramilitares y quienes se favorecieron de ellas siguen operando en las regiones, muchas de ellas organizadas para delinquir o en bandas criminales" ( p. 3).

Al considerar tanto a la escuela y la familia como agentes principales de cambio de la sociedad, el $1^{\circ}$ de septiembre de 2014 el congreso de la república de Colombia aprueba la Ley 1732 por la cual se establece la catedra de la paz y el 25 de mayo de 2015 el presidente Juan Manuel Santos firmó el decreto 1038 que reglamenta su implementación en los currículos de todas las instituciones educativas del país tanto de carácter oficial como 
privado a más tardar el 31 de diciembre de 2015. Dicha catedra tiene como objetivo fomentar el proceso de apropiación de conocimientos y competencias relacionados con la paz.

Por otra parte, Los diálogos de paz entre el gobierno nacional y las fuerzas armadas revolucionarias de Colombia FARC-EP llevados a cabo en Oslo Noruega y la Habana Cuba dan como resultado la firma de unos acuerdos de manera bilateral, que mediante el decreto 882 de 2017 se establece entre otras cosas la reglamentación para la educación en las zonas afectadas por el conflicto armado en Colombia. Lo anterior implica que algunas teorías de enseñanza, aprendizaje y procesos pedagógicos adoptados en los establecimientos educativos ubicados en dichas zonas amerite la revisión y si es el caso su re significación para la transición hacia el postconflicto.

Es por ello que el presente trabajo tiene como propósito analizar la incidencia de los procesos curriculares de las instituciones educativas ubicadas en zonas afectadas por el conflicto armado (IEZAC en adelante), en la implementación de los acuerdos de Paz y el desarrollo de la educación rural en el municipio de Montelíbano Córdoba Colombia. En consecuencia, establecer un debate que permita develar las necesidades e intereses de dichas comunidades educativas y a la luz de los hallazgos obtenidos se posibilite la configuración de los procesos curriculares para escenarios de postconflicto que redunde en la formación de ciudadanos en el saber convivir y resilientes, dispuestos a no repetir los errores de una violenta historia que les ha tocado afrontar.

\section{ESTRATEGIAS METODOLÓGICAS}

La problemática abordada se desarrolla en comunidades conformadas por padres de familia, directivos, docentes y estudiantes de la zona rural que vivieron y/o viven el abandono estatal, el horror de la guerra de guerrillas y el paramilitarismo. Por lo que se asume un enfoque cualitativo al producir datos descriptivos, las propias palabras de las personas, habladas o escritas, y la conducta observable. (Tylor y Bogdan 1987, p. 19). Desde un estudio interpretativo que pretende "encontrar pautas de relación interna en un evento para llegar a un conocimiento más profundo de éste, que la mera descripción. Identifica pautas de relación no evidente para ser usadas en investigaciones posteriores” (Hurtado, 2010).

A partir de un estudio documental cualitativo se hace la revisión de material escrito como los planes de estudio, planes de área, proyectos transversales, sistema institucional de evaluación, manuales de convivencia que convergen en el proyecto educativo institucional 
P.E.I., reciente informe técnico de visita, periódicos y revistas relacionadas con las 7 instituciones educativas rurales ubicadas en zonas afectadas por el conflicto armado en el municipio de Montelíbano Córdoba.

\section{RESULTADOS Y DISCUSIÓN}

Para hablar de procesos curriculares en las instituciones educativas ubicadas en zonas afectas por el conflicto armado, como punto de partida se hace necesario definir el término currículo, palabra que se origina del latín curriculum que significaba una pista circular de atletismo, que según Hamilton y Gibbons (1980) citado por Kemmis, S., (1993), aparece por primera vez en el ámbito educativo en países de habla inglesa en la Universidad de Glasgow, en el año 1633; como una combinación de los términos Disciplina (usado por los jesuitas desde fines del siglo XVI para referirse a un orden estructural más que secuencial) y Ratio Studiorum (esquema de estudios más que una tabla secuencial de contenidos) primitivamente usados para describir los cursos académicos en el contexto de la reforma Calvinista Escocesa ( pp.11-12)

Por su parte, Franklin Bobbitt (1876-1952), profesor de la Universidad de Wisconsin, Estados Unidos de América, mejor conocido por sus encuestas periódicas sobre evaluación de los sistemas escolares locales donde evaluó la adecuación de los planes de estudio y la publicación de sus dos libros: The Curriculum (1918) y How to make a Curriculum (1924), entre otros escritos sienta las bases de su teoría del desarrollo curricular a lo que ha llegado a llamarse el movimiento de eficiencia social en la educación y quien concibe el currículo como el rango total de experiencias, dirigidas o no, comprometido en desarrollar habilidades del individuo.

Tyler, R., (1949) define Currículo Son todas las experiencias de aprendizaje planeadas y dirigidas por la escuela para alcanzar sus metas educacionales, según Taba, H., (1962) Es el esfuerzo total de las escuelas para lograr los resultados deseados en las situaciones escolares y extraescolares. En este orden de ideas, Gagné R., (1967) sostiene que el Currículo es una secuencia de unidades de contenido arreglada de tal forma que el aprendizaje de cada unidad puede ser realizado como un acto simple. Mientras que, Stenhoue, L., (1975) lo asume como un proyecto global, integrado y flexible que muestra una alta susceptibilidad, para ser traducido en la práctica concreta instruccional. Por otro lado, Acuña, Figueroa y Díaz Barriga (1981) consideran el currículo como un Proceso dinámico de adaptación al cambio social, en general, y al sistema educativo en particular. 
Así mismo, Dewey, J., (1994) enfatizó que era un medio de transmisión sistemática de la experiencia cultural de la raza, otros valoraron su utilitarismo.

De acuerdo con lo anterior, el termino currículo resulta polisémico. Se agudiza su imprecisión al encontrar a múltiples autores ofreciendo su conceptualización que dista el uno del otro, incluso en ocasiones parecen contradictorias o disyuntivas, como se puede apreciar entre los apartes del compendio de definiciones conceptuales emitidas por diversos estudiantes de maestría y doctorado en ciencias de la educación recopiladas por Salcedo, M., y Ortiz, A., (2016), que corroboran la imprecisión de tal concepto se encuentra a Jaeson Zabala, para quien currículo es una serie de procesos encaminados a conducir mediante experiencias vividas la enseñanza y el aprendizaje de las personas, con el objetivo de desarrollar la capacidad de adaptarse a los cambios sociales mediante la solución de problemas que ayuden al desarrollo individual y colectivo así como el mejoramiento cultural para con ello lograr las metas y los objetivos de la educación. No son solo documentos físicos para la planeación instrumental de programas de estudio (...) Contrario a lo anterior, para Gustavo Bedoya, currículo son los documentos de una institución, escritos y hablados, organizados de manera sistemática y particular, que se elabora manera de consenso con la comunidad educativa a partir de una necesidad sentida, con el fin de armonizar el ambiente escolar, señalando el camino para el logro de objetivos, apoyados en la pedagogía y la didáctica como herramientas imprescindibles, teniendo en cuenta el contexto, ubicación y énfasis del plantel educativo. (pp. 31-32).

Las escuelas en aras de cumplir con su encargo social y como institución con un régimen especial, fundamentada en las ciencias y/o disciplinas auxiliares de la educación como piso para la planeación, implementación y evaluación de los procesos formativos que allí se desarrollan hacen uso de múltiples recursos e instrumentos que concurren en lo que se ha denominado Currículo, que sin la intención de abonar el universo conceptual de dicho término este debe ser entendido desde la legislación colombiana como " el conjunto de criterios, planes de estudio, programas, metodologías, y procesos que contribuyen a la formación integral y a la construcción de la identidad cultural nacional, regional y local, incluyendo también los recursos humanos, académicos y físicos para poner en práctica las políticas y llevar a el Proyecto Educativo Institucional” (Art, 76 de la Ley 115 de 1994)

No obstante, en la cotidianidad se encuentran la supremacía del currículo real por encima del currículo oficial, puesto que en las escuelas rurales especialmente en aquellas que en 
pleno siglo XXI aún los gobiernos de turnos no han llevado la energía eléctrica y por obvias razones se infiere que hay carencia de otros elementos básicos por lo que el docente además de recurrir al conocimiento didáctico del contenido, deben ser recursivo para recurrir a lo primero que encuentre en su entorno en medio de un campo abandonado a su suerte donde abunda la pobreza. Ante tal deuda de la sociedad moderna, contemporánea, analógica y digital con la ruralidad, surge el interrogante ¿Será que los avances logrados en materia educativa y teorías pedagógicas brinda los instrumentos necesarios para el desarrollo rural desde las escuelas?

Por otra parte, la historia de Colombia desde hace cinco décadas está estrechamente ligada al termino conflicto que para efectos del presente artículo se debe asumir como una condición inherente a cualquier dinamica social, dicho concepto es la fuente para discernir como se debe modelar las características primordiales en el quehacer pedagógico de las escuelas focalizadas que les ha tocado vivirlo. Ante el interrogante: ¿qué es conflicto?, los intentos por lograr una mejor aproximación conceptual han dado origen a un amplio espectro de definiciones, se reducen al tipo de conflicto que se produce entre dos o más partes o unidades sociales -individuos, grupos, organizaciones u otras unidades sociales para estar en correspondencia con el problema que nos atañe, por lo que entre algunas de las definiciones más usuales se encuentran:

- Lewis A. Coser (1956): define el conflicto como una lucha por los valores, por los bienes escasos, la potencia y el estatus, lucha en la que el objetivo de los antagonistas es el neutralizar, perjudicar o eliminar al otro.

- Raymond Aron (1964): cataloga el conflicto como una oposición entre grupos e individuos por la posesión de bienes escasos o la realización de valores mutuamente incompatibles.

- Morton Deutsch (1973): "Un conflicto existe cuando ocurren actividades incompatibles. Una actividad incompatible impide o interfiere con la ocurrencia o efectividad de una segunda actividad. Estas actividades pueden tener su origen en el interior de una persona, grupo o sociedad, o bien entre individuos, grupos o sociedades".

- Hubert Touzard (1977): el conflicto parece definir una situación en la cual unas entidades sociales apuntan a metas opuestas, afirman valores antagónicos o tienen intereses divergentes. 
- Thomas, K., (1992): Es el proceso que comienza cuando una parte percibe que la otra afecta negativamente o está próxima a afectar negativamente a algo que le concierne

- Jeffrey Z. Rubin, Dean G. Pruitt y Sung Hee Kim (1994): “divergencia percibida de intereses, o una creencia de que las aspiraciones actuales de las partes no pueden ser alcanzadas simultáneamente".

- Van de Vliert, E., (1998): Se dice que dos individuos, un individuo y un grupo o dos grupos están en conflicto en tanto que al menos una de las partes siente que está siendo obstruido o irritado por la otra.

Resulta relevante la investigación realizada en torno a la teoría de conflictos del sociólogo y matemático noruego Johan Galtung quien identifica dos situaciones clásicas como formación elemental de un conflicto: 1. Disputa: dos personas, o actores, que persiguen un mismo fin que escasea, y 2. Dilema: una persona, o actor, que persiguen dos fines incompatibles entre sí. Además, proporciona un concepto bastante elaborado de Conflicto definiéndolo como una construcción tríadica: donde observamos conductas conflictivas (B), lo que está oculto como presunciones (cogniciones) y como actitudes (emociones) agrupándolos bajo la letra A. Después tenemos el contenido del conflicto que podemos asumir que es una contradicción, C. La contradicción tiene que implicar algo deseado. Que a su estilo como buen matemático lo sintetiza como: Conflicto $=$ actitudes/presunciones + comportamiento + contradicción/contenido, es decir, Conflicto = A + B +C. (Galtung, J., 2003, PP. 107-109)

La llegada de la Catedra de la Paz incluida en los planes de estudio de todas las instituciones educativas Colombianas tanto de carácter oficial como privado a partir del 31 de diciembre de 2015 (Ley 1732 de $1^{\circ}$ de septiembre de 2014), tal como lo dispone el artículo $1^{\circ}$ del decreto 1038 del 25 de mayo de 2015, proporciona la definición de objetivos, temas y contenidos de reflexión, áreas fundamentales susceptibles para su asimilación, mecanismos para su implementación, evaluación y articulación con el sistema nacional de convivencia $¿$ acaso esta solicitud imperativa constituye un reconociendo del rol transformador social de la escuela? ¡En hora buena!

El acuerdo final para la terminación del conflicto y la construcción de una paz estable y duradera firmado por el Gobierno Nacional FARC-EP en el punto 1 numeral 1.3.2.2 el Gobierno Nacional se compromete a crear e implementar el Plan Especial de Educación Rural (PEER) con el propósito de brindar atención integral a la primera infancia, garantizar 
la cobertura, la calidad y la pertinencia de la educación y erradicar el analfabetismo en las áreas rurales, así como promover la permanencia productiva de los y las jóvenes en el campo, y acercar las instituciones académicas regionales a la construcción del desarrollo rural. (Acuerdo final para la terminación del conflicto y la construcción de una paz estable y duradera, 2016, p. 26)

La propuesta curricular de cada establecimiento educativo, debe describir las acciones que se llevan a cabo para la formación integral de sus educandos. En tal sentido, los planes de estudio, planes de área, proyectos transversales, sistema institucional de evaluación, Manual de convivencia, entre otros documentos obligatorios convergen en el Proyecto Educativo Institucional PEI. Razón por la cual se realizó una revisión por los componentes de dicho Proyecto, así como al más reciente informe técnico realizado a las instituciones focalizadas en el municipio de Montelíbano Córdoba, con el propósito de analizar y reflexionar acerca de los posibles aportes de los procesos formativos en lo que al punto 1 numeral 1.3.2.2 del acuerdo final de paz entre el gobierno y la FARC-EP se refiere. En consecuencia, se plantea el siguiente interrogante, ¿Qué incidencia tienen los procesos curriculares de las escuelas ubicadas en zonas afectadas por el conflicto armado, en la implementación de los acuerdos de Paz y el desarrollo de la educación rural en la subregión de Córdoba Colombia? El resultado de dicha revisión se presenta en el siguiente cuadro.

Cuadro $N^{\circ} 1$. Estrategias del PEER articuladas con el PEI.

\begin{tabular}{|c|c|c|c|c|c|c|}
\hline Código & $\begin{array}{l}\text { ¿Qué } \\
\text { estrategias } \\
\text { implementa la } \\
\text { I.E. para } \\
\text { favorecer la } \\
\text { continuidad de } \\
\text { los niños, } \\
\text { niñas, } \\
\text { adolescentes y } \\
\text { jóvenes en su } \\
\text { trayectoria } \\
\text { educativa? }\end{array}$ & $\begin{array}{l}\text { ¿De qué } \\
\text { manera se } \\
\text { promueve } \\
\text { la } \\
\text { participació } \\
\text { n de las } \\
\text { familias y } \\
\text { otros } \\
\text { actores de } \\
\text { la } \\
\text { comunidad } \\
\text { en los } \\
\text { procesos } \\
\text { formativos? }\end{array}$ & $\begin{array}{l}\text { ¿Qué acciones } \\
\text { se llevan a cabo } \\
\text { para la } \\
\text { promoción de la } \\
\text { Educación para } \\
\text { la paz, la } \\
\text { reconciliación y } \\
\text { la convivencia? }\end{array}$ & $\begin{array}{l}\text { ¿La I.E. está } \\
\text { vinculada a } \\
\text { programas } \\
\text { alfabetizació } \\
\mathrm{n} \text { y } \\
\text { continuidad } \\
\text { de la la } \\
\text { educación } \\
\text { formal de } \\
\text { adultos? En } \\
\text { caso } \\
\text { afirmativo } \\
\text { diga cuál(es) }\end{array}$ & $\begin{array}{l}\text { ¿El plan de } \\
\text { estudio } \\
\text { contempla la } \\
\text { incorporació } \\
\mathrm{n} \text { de la } \\
\text { formación } \\
\text { técnica } \\
\text { agropecuaria } \\
\text { en el nivel de } \\
\text { educación } \\
\text { media? }\end{array}$ & $\begin{array}{l}\text { ¿La I.E. } \\
\text { brinda } \\
\text { algún } \\
\text { Modelo } \\
\text { Educativo } \\
\text { Flexible? } \\
\text { En caso } \\
\text { afirmativo } \\
\text { diga cuál. }\end{array}$ \\
\hline IESIMBOL & $\begin{array}{l}\text { Matricula } 0 . \\
\text { Restaurante } \\
\text { escolar }\end{array}$ & $\begin{array}{l}\text { Consejo } \\
\text { directivo, } \\
\text { junta y } \\
\text { Asamblea } \\
\text { de padres }\end{array}$ & $\begin{array}{lr}\text { Proyecto } & \\
\text { transversal } & \text { de } \\
\text { democracia } & y \\
\text { comité } & \text { de } \\
\text { convivencia }\end{array}$ & $\begin{array}{l}\text { Actualmente } \\
\text { ninguno }\end{array}$ & $\mathrm{Si}$ & $\begin{array}{c}\text { Escuela } \\
\text { nueva }\end{array}$ \\
\hline
\end{tabular}




\begin{tabular}{|c|c|c|c|c|c|c|}
\hline IEPAL & $\begin{array}{l}\text { Matricula } 0 . \\
\text { Restaurante } \\
\text { escolar }\end{array}$ & $\begin{array}{l}\text { Consejo } \\
\text { directivo, } \\
\text { junta y } \\
\text { Asamblea } \\
\text { de padres }\end{array}$ & $\begin{array}{ll}\text { Proyecto } & \\
\text { transversal } & d \\
\text { democracia } & \\
\text { comité } & d \\
\text { convivencia } & \\
\end{array}$ & $\begin{array}{l}\text { Actualmente } \\
\text { ninguno }\end{array}$ & No & $\begin{array}{c}\text { Escuela } \\
\text { nueva }\end{array}$ \\
\hline IETAC & $\begin{array}{ll}\text { Matricula } & 0 . \\
\text { Restaurante } & \\
\text { escolar, } & \\
\text { proyecto } & \\
\text { cultural } & \end{array}$ & $\begin{array}{l}\text { Consejo } \\
\text { directivo, } \\
\text { comité de } \\
\text { evaluación } \\
\text { y } \\
\text { promoción, } \\
\text { junta y } \\
\text { Asamblea } \\
\text { de padres, } \\
\text { proyecto } \\
\text { productivo } \\
\text { granja } \\
\text { escolar. }\end{array}$ & $\begin{array}{l}\text { Proyecto } \\
\text { transversal } \\
\text { democracia } \\
\text { comité } \\
\text { convivencia }\end{array}$ & $\begin{array}{l}\text { Actualmente } \\
\text { ninguno }\end{array}$ & $\mathrm{Si}$ & $\begin{array}{c}\text { Escuela } \\
\text { nueva }\end{array}$ \\
\hline IEJOMAC & $\begin{array}{l}\text { Matricula } 0 . \\
\text { Restaurante } \\
\text { escolar }\end{array}$ & $\begin{array}{l}\text { Consejo } \\
\text { directivo, } \\
\text { junta y } \\
\text { Asamblea } \\
\text { de padres }\end{array}$ & $\begin{array}{l}\text { Proyecto } \\
\text { transversal } \\
\text { democracia } \\
\text { comité } \\
\text { convivencia }\end{array}$ & $\begin{array}{l}\text { Actualmente } \\
\text { ninguno }\end{array}$ & No & $\begin{array}{c}\text { Escuela } \\
\text { nueva }\end{array}$ \\
\hline IESANFRA & $\begin{array}{l}\text { Matricula } 0 . \\
\text { Restaurante } \\
\text { escolar }\end{array}$ & $\begin{array}{l}\text { Consejo } \\
\text { directivo, } \\
\text { junta y } \\
\text { Asamblea } \\
\text { de padres } \\
\end{array}$ & $\begin{array}{ll}\text { Proyecto } & \\
\text { transversal } & d \\
\text { democracia } & \\
\text { comité } & d \\
\text { convivencia }\end{array}$ & $\begin{array}{l}\text { Actualmente } \\
\text { ninguno }\end{array}$ & No & $\begin{array}{c}\text { Escuela } \\
\text { nueva }\end{array}$ \\
\hline IESANJO & $\begin{array}{l}\text { Matricula } 0 . \\
\text { Restaurante } \\
\text { escolar }\end{array}$ & $\begin{array}{l}\text { Consejo } \\
\text { directivo, } \\
\text { junta y } \\
\text { Asamblea } \\
\text { de padres }\end{array}$ & $\begin{array}{ll}\text { Proyecto } & \\
\text { transversal } & d \\
\text { democracia } & \\
\text { comité } & d \\
\text { convivencia } & \end{array}$ & & No & $\begin{array}{c}\text { Escuela } \\
\text { nueva }\end{array}$ \\
\hline INESAN & $\begin{array}{l}\text { Matricula } 0 . \\
\text { Restaurante } \\
\text { escolar }\end{array}$ & $\begin{array}{l}\text { Consejo } \\
\text { directivo, } \\
\text { junta y } \\
\text { Asamblea } \\
\text { de padres }\end{array}$ & $\begin{array}{l}\text { Proyecto } \\
\text { transversal } \\
\text { democracia } \\
\text { comité } \\
\text { convivencia }\end{array}$ & & No & $\begin{array}{c}\text { Escuela } \\
\text { nueva }\end{array}$ \\
\hline
\end{tabular}

Fuente: adaptado del PEER (2018)

De lo anterior, se observa que una de las 7 instituciones educativas del municipio de Montelíbano de carácter oficial, ubicada en zona rural y focalizada por la entidad territorial certificada Córdoba por estar ubicada en zona afectada por el conflicto armado, que además de permitir la participación de padres de familia y organizaciones de los mismos en la construcción y actualización del PEI, gobierno escolar y planes de mejoramiento, vincula anualmente a un núcleo familiar para que resida en los predios de la institución (25 Hectáreas) y a su vez instruyan a los estudiantes que cursan los grados noveno, decimo y undécimo en a la actividad agrícola, ganadera y cría de especies menores, siendo esta la 
IETAC y junto a la IESIMBOL mantienen convenios con el SENA para formar a sus educando de educación media en producción agropecuaria y piscicultura, dicha propuesta curricular está alineada con la estrategia 1. Fortalecimiento de la participación familiar y comunitaria en la Gestión Educativa (PEER, 2018. P. 73)

Así mismo, es relevante anotar que la primera Institución antes mencionada dispone de un proyecto cultural denominado $A l$ son de pitos y tambores que realiza sus actividades en jornada contraria para mantener a los niños y jóvenes alejados del ocio y otros distractores, así como una escuela de fundamentación deportiva estrellas de la paz y la banda rítmica que lleva el nombre del establecimiento educativo que ameniza las fiesta cívicas y jornadas culturales en la institución y las aledañas acciones estrechamente ligadas a la Estrategia 4. Oferta de aulas abiertas culturales y deportivas para la comunidad donde evidentemente la escuela trasciende más allá de las aulas o salones para incursionar en espacios comunitarios para desarrollar procesos de formación cultural, académica, recreativa y deportiva. (PEER, 2018, P. 81)

Por otro lado, resulta curioso observar que paradójicamente las instituciones con mayor afectación por el conflicto armado, donde actuaron y actúan grupos armados al margen de la ley (tanto guerrilla como paramilitares), no se implemente la catedra de la paz, o cualquiera otra estrategia que le permita realizar un trabajo pedagógico diferenciado con sus estudiantes y enfocarlos hacia la resolución de conflictos de forma pacífica más allá de lo dispuesto en las rutas de los manuales de convivencia y el área de ciencias sociales.

Finalmente, a través de la revisión documental realizada se cuenta con elementos orientadores para indicar que la relación entre las estrategias del PEER para la Educación inicial, preescolar, básica y media que atienden las dimensiones: escuela, familia y comunidad, académica y pedagógica, docentes y directivos docentes, administrativa, ambiente escolar y bienestar frente a las propuestas curriculares de las instituciones educativas focalizadas no se encuentran lo suficientemente alineadas; lo que invita a realizar una revisión y actualización de los manuales de convivencia, proyectos transversales y productivos, planes de estudio, entre otros que conforman el PEI que posibilite mayores aportes de las mismas en la construcción de una paz estable y duradera.

\section{CONSIDERACIONES FINALES}

La Ley General de Educación, que otorga la autonomía escolar dispone entre otros apartes que “...las instituciones de educación formal gozan de autonomía para organizar las áreas 
fundamentales definidas para cada nivel, introducir asignaturas optativas dentro de las áreas establecidas en la ley, adaptar algunas áreas a las necesidades y características regionales, adoptar métodos de enseñanza...” (Ley 115 de 1994, art. 77)

Así mismo, En lo concerniente a la educación campesina y rural, se encuentra que:

"El Gobierno Nacional y las entidades territoriales promoverán un servicio de educación campesina y rural la cual comprenderá especialmente la formación técnica en actividades agrícolas, pecuarias, pesqueras, forestales y agroindustriales que contribuyan a mejorar las condiciones humanas, de trabajo y la calidad de vida de los campesinos y a incrementar la producción de alimentos en el país”. (Ley 115 de 1994, art. 64)

En este sentido, los procesos curriculares de las Instituciones Educativas ubicadas en Zonas Afectadas por el conflicto Armado en el municipio de Montelibano Córdoba que en su totalidad se encuentran en la ruralidad y considerando su nuevo tipificación, se hace necesario la revisión de su diseño curricular, en particular en lo que a sus procesos pedagógicos se refiere, para reflexionar si responden o no, y de qué manera a las nuevas expectativas durante la transición del conflicto al postconflicto, formando niños y jóvenes en la tolerancia y resiliencia, como gestores de paz, técnicos agropecuarios o agroindustriales, así como amigables con el ambiente.

Sin embargo, se puede apreciar que las propuestas curriculares de las instituciones focalizadas adoptan e implementan modelos pedagógicos que resultan ser adaptaciones de modelos foráneos o extranjeros, entre ellos, Holístico, Humanista o Humanistacristiano-Constructivista. Por lo que vale la pena cuestionar si las teorías de enseñanza, aprendizaje y evaluativas allí empleadas no abordan la problemática social que vive la comunidad educativa rural en el siglo XXI.

Además, en los planes de estudio, área y/o de aula de dichas instituciones se evidencia un diseño curricular por competencias donde prima la formación para el trabajo, la modalidad académica y la medición de la calidad de los aprendizajes de los estudiantes en función de los puntajes obtenidos en las pruebas internas y externas, tan solo 2 de las 7 instituciones focalizadas mantienen convenio con el Servicio Nacional de Aprendizaje para la formación técnica de sus estudiantes en producción agropecuaria y piscicultura como una apuesta estructural en la transformación del niño o niña como sujeto social y emocional. 
En consecuencia, los diseños curriculares de las escuelas ubicadas en zonas afectas por el conflicto armado en Colombia, particularmente las focalizadas en el municipio de Montelibano Córdoba, abordan contenidos a juicio del docente amparados en la libertad de cátedra y en el mejor de los casos se limitan a desarrollar los aprendizajes y competencias propuestas en los documentos de referencia y orientadores que propone el M.E.N. sin tener en cuenta el Plan Especial de Educación Rural consagrado en el punto 1.3.2.2. del acuerdo final de paz entre el gobierno nacional y las fuerzas armadas revolucionarias de Colombia FARC-EP. ¿Las comunidades educativas de Montelíbano Córdoba conformadas por campesinos, grupos étnicos como los Embera Katíos y grupos afrodescendientes que ahora asumen la nueva tipificación de estar ubicadas en zonas afectadas por el conflicto armado entre el estado colombiano y la FARC-EP, pueden despertar la mañana siguiente y exclamar "Cesó la horrible"?

\section{LISTA DE REFERENCIAS}

Bautista, N. (2011). Proceso de la investigación cualitativa: Epistemología, metodología y aplicaciones. Ed. El Manual Moderno, Bogotá ISBN: 978-958.9446-46-1.

Congreso de la República de Colombia. (2016, 12 de noviembre). Acuerdo Final para la Terminación del Conflicto y la Construcción de una Paz Estable y Duradera. http://www.centrodememoriahistorica.gov.co/descargas/finAcuerdoPazAgosto2016 112-11-2016-Nuevo-Acuerdo-Final.pdf

Congreso de la República de Colombia. (Ley 115 del 08 de febrero de1994). Ley general de educación.

Congreso de la República de Colombia. (Ley 1732 del 01 de septiembre de 2014). Catedra de la Paz.

Galtung, J., (2003) Paz por medios pacíficos. Paz y conflicto, desarrollo y civilización, Bilbao, Gernika Gogoratuz. https://www.gernikagogoratuz.org/wpcontent/uploads/2020/05/RG07completo-A4.pdf

Katayama, R., (2014). Introducción a la investigación cualitativa: fundamentos, métodos, estrategias y técnicas. Universidad Inca Garcilaso de la vega. ISBN: 978-612-4050$75-6$

Institución Educativa Técnico Agropecuario Claret. (2015). Proyecto Educativo Institucional. Montelíbano. 
Institución Educativa El Palmar. (2015). Proyecto Educativo Institucional. Montelíbano. Institución Educativa Simón Bolívar. (2015). Proyecto Educativo Institucional. Montelíbano.

Institución Educativa José Maria Córdoba. (2015). Proyecto Educativo Institucional. Montelíbano.

Institución Educativa San Francisco del Rayo. (2015). Proyecto Educativo Institucional. Montelíbano.

Institución Educativa San José. (2015). Proyecto Educativo Institucional. Montelíbano. Institución Educativa Dulce Nombre de Jesus. (2015). Proyecto Educativo Institucional. Montelíbano.

Kemmis, S. (1993). El currículum más allá de la teoría de la Reproducción. 2ed, Madrid: Morata.

Ministerio de educación nacional M.E.N. (2018). Plan especial de educación rural hacia el desarrollo rural y la construcción de paz https://www.mineducacion.gov.co/1759/articles-385568_recurso_1.pdf

Ministerio de agricultura y desarrollo rural (decreto 893 de 28 de mayo de 2017). Programa de Desarrollo con Enfoque Territorial- PDET

SED Córdoba (2020). Informe técnico de visita a establecimiento educativo oficial de preescolar, básica y media para ampliación del servicio, I.E.T.A.C Montelibano

SED Córdoba (2020). Informe técnico de visita a establecimiento educativo oficial de preescolar, básica y media para ampliación del servicio, I.E. El Palmar, Montelibano SED Córdoba (2020). Informe técnico de visita a establecimiento educativo oficial de preescolar, básica y media para ampliación del servicio, I.E. Simón Bolívar, Montelibano

SED Córdoba (2020). Informe técnico de visita a establecimiento educativo oficial de preescolar, básica y media para ampliación del servicio, I.E. José Maria Córdoba, Montelibano

SED Córdoba (2020). Informe técnico de visita a establecimiento educativo oficial de preescolar, básica y media para ampliación del servicio, I.E. San Francisco del Rayo, Montelibano

SED Córdoba (2020). Informe técnico de visita a establecimiento educativo oficial de preescolar, básica y media para ampliación del servicio, I.E. San José, Montelibano 
SED Córdoba (2020). Informe técnico de visita a establecimiento educativo oficial de preescolar, básica y media para ampliación del servicio, I.E. Antoni Nariño, Montelibano

Taylor, S., y Bogdan, R., (1987). Introducción a los métodos cualitativos de investigación: La busqueda de significados. Ediciones Paidós Iberica S.A. Buenos Aires ISBN: 84-7509-816-9 